\title{
A Fhit-mimetic peptide suppresses annexin A4-mediated chemoresistance to paclitaxel in lung cancer cells
}

\author{
Eugenio Gaudio ${ }^{1,2,3, *}$, Francesco Paduano ${ }^{3, *}$, Apollinaire Ngankeu ${ }^{1}$, Francesco \\ Ortuso ${ }^{4}$, Francesca Lovat ${ }^{1}$, Sandra Pinton ${ }^{2}$, Sabrina D'Agostino ${ }^{3}$, Nicola Zanesi ${ }^{1}$, \\ Rami I. Aqeilan ${ }^{1,5}$, Pietro Campiglia', Ettore Novellino7, Stefano Alcaro ${ }^{4}$, Carlo M. \\ Croce $^{1}$ and Francesco Trapasso ${ }^{3}$ \\ ${ }^{1}$ Department of Molecular Immunology, Virology and Medical Genetics, The Ohio State University, Columbus, Ohio, USA \\ ${ }^{2}$ Lymphoma \& Genomics Research Program, IOR Institute of Oncology Research, Bellinzona, Switzerland \\ ${ }^{3}$ Dipartimento di Medicina Sperimentale e Clinica, University Magna Græcia, Campus "S. Venuta", Catanzaro, Italy \\ 4 Dipartimento di Scienze della Salute, University Magna Græcia, Campus "S. Venuta", Catanzaro, Italy \\ ${ }^{5}$ The Lautenberg Center for Immunology and Cancer Research, Institute for Medical Research, The Hebrew University, \\ Jerusalem, Israel \\ ${ }^{6}$ Dipartimento di Farmacia, Università di Salerno, Fisciano, Italy \\ 7 Dipartimento di Farmacia, Università degli Studi di Napoli "Federico II", Napoli, Italy \\ * These authors have contributed equally to this work \\ Correspondence to: Carlo M. Croce, email: carlo.croce@osumc.edu \\ Francesco Trapasso, email: trapasso@unicz.it
}

Keywords: fragile histidine triad, FHIT, annexin A4, ANXA4, mimetic peptides

Received: January 07, $2016 \quad$ Accepted: April 11, $2016 \quad$ Published: May 04, 2016

ABSTRACT

We recently reported that Fhit is in a molecular complex with annexin A4 (ANXA4); following to their binding, Fhit delocalizes ANXA4 from plasma membrane to cytosol in paclitaxel-resistant lung cancer cells, thus restoring their chemosensitivity to the drug. Here, we demonstrate that Fhit physically interacts with A4 through its $\mathrm{N}$-terminus; molecular dynamics simulations were performed on a 3D Fhit model to rationalize its mechanism of action. This approach allowed for the identification of the QHLIKPS heptapeptide (position 7 to 13 of the wild-type Fhit protein) as the smallest Fhit sequence still able to preserve its ability to bind ANXA4. Interestingly, Fhit peptide also recapitulates the property of the native protein in inhibiting Annexin A4 translocation from cytosol to plasma membrane in A549 and Calu-2 lung cancer cells treated with paclitaxel. Finally, the combination of Tat-Fhit peptide and paclitaxel synergistically increases the apoptotic rate of cultured lung cancer cells and blocks in vivo tumor formation.

Our findings address to the identification of chemically simplified Fhit derivatives that mimic Fhit tumor suppressor functions; intriguingly, this approach might lead to the generation of novel anticancer drugs to be used in combination with conventional therapies in Fhit-negative tumors to prevent or delay chemoresistance.

\section{INTRODUCTION}

The FHIT gene encompasses the most common fragile site, $F R A 3 B$ at 3 p14.2 $[1,2]$ in human lymphoblasts; interestingly, although this region is not the most fragile locus in epithelial cells [3], FHIT loss is commonly reported in human tumors of this derivation as an early event [4], not only through genetic inactivation (i.e., deletions and translocations) [5] but also through the silencing of its promoter due to hypermethylation [6]. 
Several evidences pointed at FHIT as a tumor suppressor gene; in fact, its genetic ablation in mice results both in an increase of spontaneous tumors and in a much higher susceptibility to develop carcinogeninduced tumors than wild-type counterparts [7]. Moreover, FHIT overexpression driven in vivo through recombinant viral vectors, prevented tumor development in Fhit knock-out mice [8, 9]. Furthermore, FHIT restoration in FHIT-negative cancer cells of both epithelial origin and leukemias blocks in vivo tumor formation and triggers caspase-mediated apoptosis [10-12].

FHIT is one of the genes mostly involved in human tumorigenesis; however, regardless of the efforts, Fhit function still remains largely obscure. FHIT encodes for an enzyme whose in vivo substrate has not been identified yet; however, in cell free assays its product hydrolizes diadenosine polyphosphate substrates, preferentially diadenosine triphosphate (Ap3A) but also diadenosine tetraphosphate (Ap4A) [13, 14]. The design of mutant alleles of FHIT proved that only mutants still binding the substrate could, at least partially, retain the ability to trigger apoptosis of Fhit-negative cancer cells $[15,16]$. Only recently, we begun to shed lights about the molecular pathways involving Fhit protein activity. In fact, Hsp60 and Hsp10 take Fhit protein into the mitochondrion where it binds some mitochondrial proteins, including Fdxr; this protein complex induces the generation of reactive oxygen species (ROS) which, in turn, triggers apoptosis of Fhitnegative cancer cells [17]. As Fhit protein is virtually ubiquitously distributed into the cell [17, 18], later on we tried to isolate novel Fhit partners with oncogenic activity starting from cell membranes; we successfully identified annexin A4 (ANXA4) [19], a protein belonging to a superfamily of calcium-regulated and phospholipid membrane-binding proteins [20]. ANXA4 is overexpressed in various tumors [21-23] and it is involved in chemoresistance [21, 22, 24-27] other than invasion and metastasis [28]. Intriguingly, Fhit restoration in Fhitnegative lung cancer cells blocks ANXA4 translocation from cytosol to the inner side of plasma membrane during paclitaxel administration, an effect that contribute to chemoresistance, thus sensitizing again cancer cells to the drug [19].

In this study, we report that a short Fhit-derived peptide still able to physically bind ANXA4, recapitulates the Fhit wild-type activity with regard to ANXA4, as this Fhit sequence can prevent ANXA4 translocation to plasma membrane in paclitaxel-treated lung cancer cells, thus resulting in the restoration of chemosensibility to the drug both in vitro and in vivo experiments.

\section{RESULTS}

\section{Annexin 4 binds the $\mathbf{N}$-terminus of Fhit protein}

In order to map the region of Fhit protein binding Annexin A4, we cloned downstream GST three truncated FHIT cDNAs, namely TR1 (332 bp), TR2 (212 bp) and TR3 (113 bp), all containing the N-terminus common to the wild-type Fhit protein. TR1, TR2, and TR3 correspond to the 111,71 , and $38 \mathrm{~N}$-terminal aminoacids of Fhit protein, respectively (Figure 1A). Annexin A4 binds wild-type Fhit/GST fusion protein as well as all shorter forms of Fhit fusion proteins, indicating its binding to the $\mathrm{N}$-terminus of Fhit (Figure 1B). In our previous paper, we reported that Fhit/Annexin A4 interaction was only detectable in the presence of DSP [19]. DSP is a cross-linker containing an amine-reactive N-hydroxysuccinimide (NHS); NHS esters react with primary amine group located at lysine (Lys, K) residue sidechain. Since Lys residues are abundant and easily accessible on the hydrophilic surface of most proteins they usually crosslink with high efficiency.

We assumed that the Lys interacting to DSP must be positioned on a Fhit surface region binding Annexin A4. Due to the unavailability of experimental structures reporting such a complex, molecular modeling studies were performed to highlight the most favored Lys residues for interacting with DSP (Supplementary Figure S1). Taking into account the reaction between the Lys and the cross-linker, we have considered two different molecular descriptors in order to predict the most favored adduct formation: a) the exposition of the nucleophilic residue, and b) the electron availability on the primary amine located at the Lys sidechain. The first descriptor was computed as the average solvent surface area (ASASA) related to the Lys sidechain and the second was derived from the residue partial charge distribution. Both parameters required to take into account the Fhit conformation flexibility; consequently extensive molecular dynamics (MD) simulations were performed on our Fhit 3D model sampling, at regular time intervals, 150 structures. These conformers were used for the ASASA calculation and, after clusterisation, for the quantum mechanics computing of the Mulliken charge distribution (Supplementary Figures S2-S3). Moreover, with the aim to deep evaluate Fhit conformational properties, in particular highlighting the most flexible protein areas, MD trajectory was submitted to residue fluctuation analysis (RFA) (Supplementary Figure S4). The results marked the Fhit 108-127 loop as having greater flexibility than the remainder of the protein. Taking into account its experimental activity, we focused our attention on structural similarities between the Fhit peptide segment 1-38 and the full length protein, by submitting the short peptide to the same MD protocol adopted for Fhit. The MD 
trajectories of 1-38 and Fhit were compared by computing, frame by frame, the root mean square deviation (RMSd), generating a squared matrix containing 22500 RMSd values (corresponding to all possible combination among the 1-38 and Fhit MD structures). Such an analysis revealed remarkable similarities, with $\sim 81 \%$ of the structures reporting an all atoms RMSd value ranging from 2 to $4 \AA$ (Supplementary Figure S5). To identify the region of 1-38, containing Lys residues (11, 18 and 29), structurally closest to Fhit, the short peptide MD trajectory was submitted to the same RFA previously described for the full length protein. The comparison between 1-38 and Fhit RFA revealed the stronger analogies for the region containing Lys11 (Supplementary Figure S6). In particular His8, Leu9, Ile10, Lys11 and Pro12 of 1-38 peptide reported an average difference in terms of RMSd of about $0.02 \AA$ with respect to the full-length Fhit.

On the basis of the above reported information, we addressed to the Fhit 8-12 residue sequence a key role in the Annexin 4 recognition. With the aim to improve water solubility of the new shorter peptide, we included hydrophilic residues at Fhit position 7 (Gln) and 13 (Ser).

To validate this hypothesis, we tested a synthetic Fhit 7-13 peptide on A549 and Calu-2 cultured lung cancer cells. To assure the cell permeation of Fhit 7-13 peptide, the sequence was fused downstream to a Tat peptide (an epitope mapping at position 47-58 of the HIV Tat protein); $24 \mathrm{~h}$ after Tat-Fhit 7-13 peptide administration, cell lysates were co-immunoprecipitated with a Tat monoclonal antibody. As demonstrated in Figure 1C, Annexin 4 was detectable only in the presence of Fhit 7-13 peptide, but not in cells treated with a Tat-Fhit 7-13 scrambled peptide.

\section{Fhit peptide blocks annexin 4 translocation from cytosol to plasma membrane}

As previously reported [19], treatment of A549 and Calu-2 cancer cells with paclitaxel induced cytosolic depletion of Annexin A4 that underwent a considerable

\section{A}
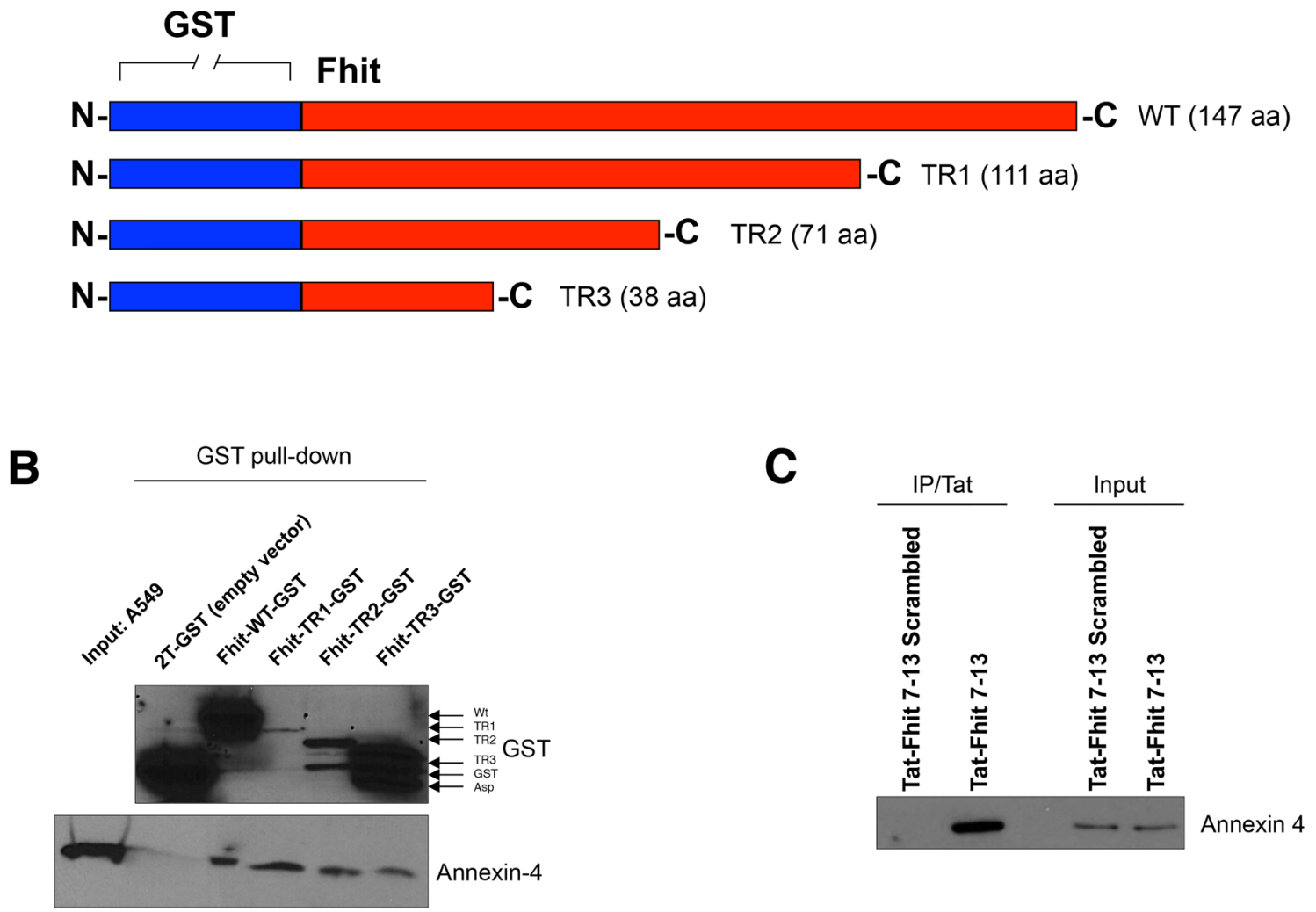

Figure 1: Fhit peptide interacts with Annexin 4. A. GST-Fhit fusion protein and three deletion mutant proteins. Fhit was deleted from the C-terminal site. B. GST-FHIT plasmids were amplified in BL21 bacteria by stimulation with IPTG $0.5 \mu \mathrm{M}$ for $6 \mathrm{~h}$ at $30^{\circ} \mathrm{C}$. Recombinant GST-Fhit fusion proteins were purified with GSH resin beads and added to A549 total lysates. $12 \mathrm{~h}$ after incubation at $4^{\circ} \mathrm{C}$, GSH resin beads were washed and proteins eluted. Proteins were separated on a polyacrylamide gel, transferred to nitrocellulose filters and probed with antibodies raised against Annexin 4 or GST. C. A549 cells were treated with Tat-scrambled peptide or Tat-Fhit 7-13 peptide $(150 \mu \mathrm{M}) 24 \mathrm{~h}$ after Tat-Fhit 7-13 peptide administration, cell lysates enriched in membrane fraction were co-immunoprecipitated with a Tat monoclonal antibody, proteins were separated on a polyacrylamide gel, transferred to nitrocellulose filters and probed with an Annexin 4 antibody. Inputs were run as control for equal immunoprecipitated protein amounts. 
translocation to the inner side of plasma membrane. Fhit overexpression blocked Annexin A4 translocation from cytosol to the plasma membrane, observed after paclitaxel administration. Here, we tested the ability of the Tat-Fhit 7-13 peptide to block Annexin 4 in the cytosol. Figure 2A shows that the simultaneous treatment with paclitaxel and Tat-Fhit 7-13 peptide, was able to block Annexin A4 translocation from cytosol to plasma membrane compared to the scrambled Tat-Fhit 7-13 used as a control in both A549 and Calu-2 cells. In addition, other peptides designed from both $\mathrm{N}$ - and C-termini of the Fhit protein, failed either to bind or sequester Annexin 4 in the cytosol (data not shown). These data indicated that the $\mathrm{N}$-terminus of Fhit is responsible for the binding with Annexin 4 and, more specifically, that the peptide sequence ranging from position 7 to 13 of Fhit is crucial for the binding.

\section{Tat-Fhit 7-13 peptide triggers apoptosis of lung} cancer cells and sensitizes them to paclitxel

To determine if Tat-Fhit 7-13 peptide could mimic the effects of the wild-type Fhit protein on A549 and Calu2 lung cancer cell lines, cells were treated with a single administration of 50,100 or $150 \mu \mathrm{M}$ Fhit Tat-7-13 peptide and $24 \mathrm{~h}$ later cell death was assessed by flow cytometry. Interestingly, we observed dose-dependent sub-G1 peaks accounting for $16 \%, 22 \%$, and $32 \%$ of cell population, for the growing doses of peptide administered to A549 cells. The Tat-Fhit 7-13 scrambled peptide had no effect in triggering cell death (Figure 2B). Furthermore, Tat-Fhit 7-13 peptide sensitizes A549 cells to paclitaxel, as shown by TUNEL assay; in fact, the percentage of apoptotic cells increased three-fold compared to controls (60\% versus 20\%) (Figure 2C). Comparable results were obtained after
A

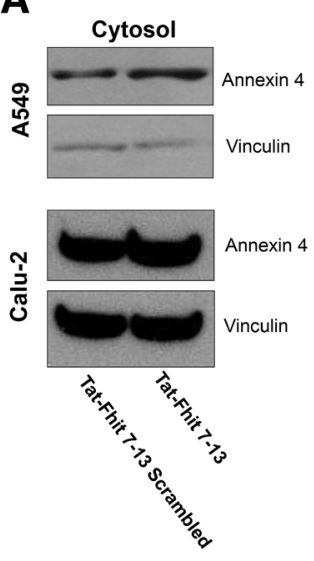

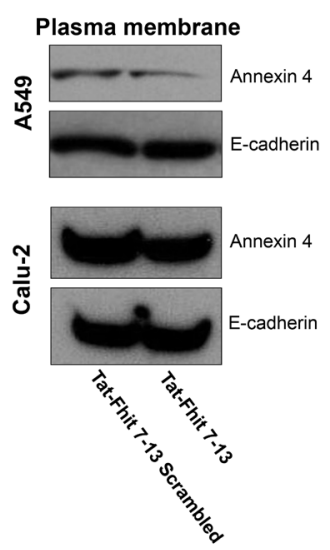

C

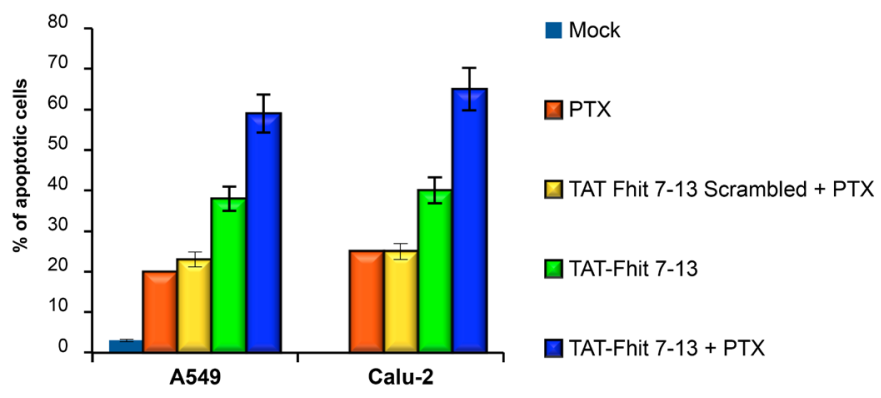

B

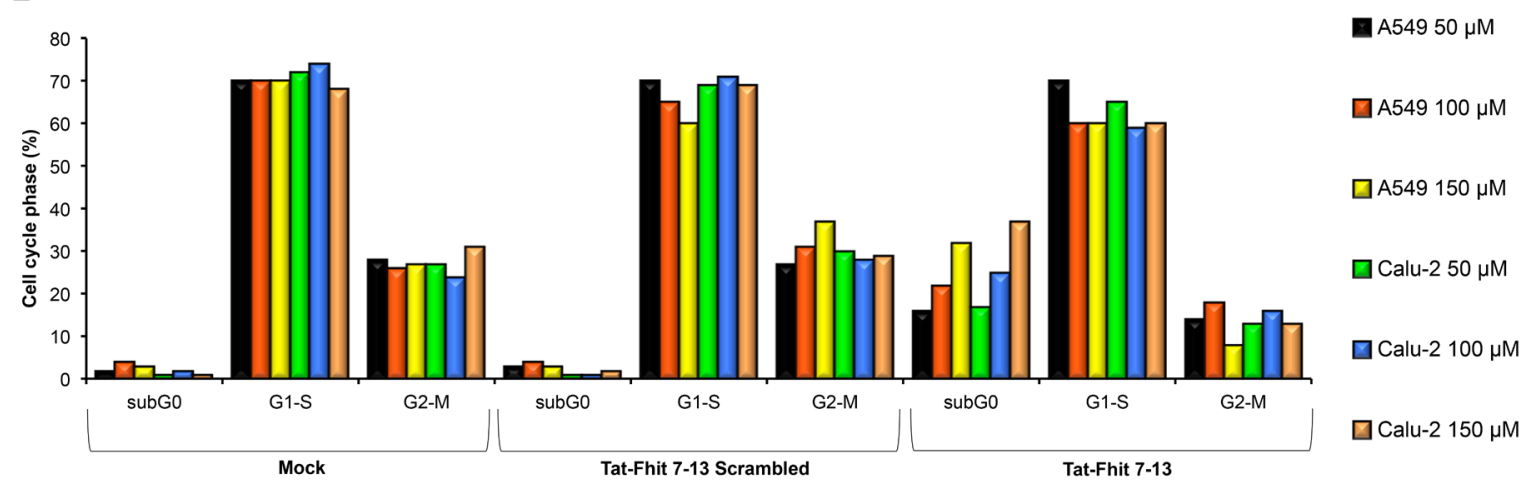

Figure 2: Tat-Fhit 7-13 peptide blocks Annexin 4 translocation from cytosol to plasma membranes, triggers apoptosis, and sensitizes lung cancer cells to paclitaxel. A. A549 and Calu-2 lung cancer cells were treated with Tat-scrambled peptide, Tat-Fhit 7-13 peptide $(150 \mu \mathrm{M})$ and $800 \mathrm{nM}$ paclitaxel for $24 \mathrm{~h}$. Proteins from cytosolic and membrane fractions were separated on a polyacrylamide gel, transferred to nitrocellulose filter, and probed with Annexin 4 antibody. Vinculin and E-cadherin were used to normalize protein loading of cytosolic and plasma membrane proteins, respectively. B. A549 and Calu-2 cells were untreated or treated with 50,100 and $150 \mu \mathrm{M}$ Tat-scrambled peptide or Tat-Fhit 7-13 peptide and then $24 \mathrm{~h}$ later evaluated by flow cytometric analysis; a representative experiment is reported. C. A549 and Calu-2 cells treated with Tat-scrambled peptide and Tat-Fhit 7-13 peptide for 24 h, as described in B, were analyzed by TUNEL assay; mean values \pm SD of three experiments are reported. 
treatment of Calu-2 cells with Tat-Fhit 7-13 peptide alone or in combination with paclitaxel (Figure 2B and 2C).

Taken together, these data indicate that the Fhit 7-13 peptide can partly recapitulate wild-type Fhit activity in triggering apoptosis of A549 and Calu-2 lung cancer cells.

\section{Tat-Fhit 7-13 peptide in combination with paclitaxel blocks in vivo tumor formation}

We tested the stability of our peptides in the culture medium over adherent cells through Mass SpectroemtryMALDI. Twenty-four hours after their administration to the medium, both scrambled and 7-13 Fhit peptides resulted still stable and undegraded (see Supplementary Figure S7A-S7C).

We further investigated the in vivo effects of TatFhit 7-13 peptide with or without paclitaxel treatment in a preclinical model of lung cancer, using six groups of mice ( $n=5$ mice per group). Each group was subcutaneously injected with $1 \times 10^{7}$ A549 cells. When tumors reached the diameter of $15 \mathrm{~mm}$, three groups were treated either with a single administration of $40 \mathrm{mg} / \mathrm{kg}$ paclitaxel, or $50 \mathrm{mg} /$ kg Tat-Fhit 7-13 peptide, or both; mice were monitored on a regular basis. The remaining three groups, used as controls, were either left untreated or treated with Tat-Fhit 7-13 scrambled or treated with Tat-Fhit 7-13 scrambled
A

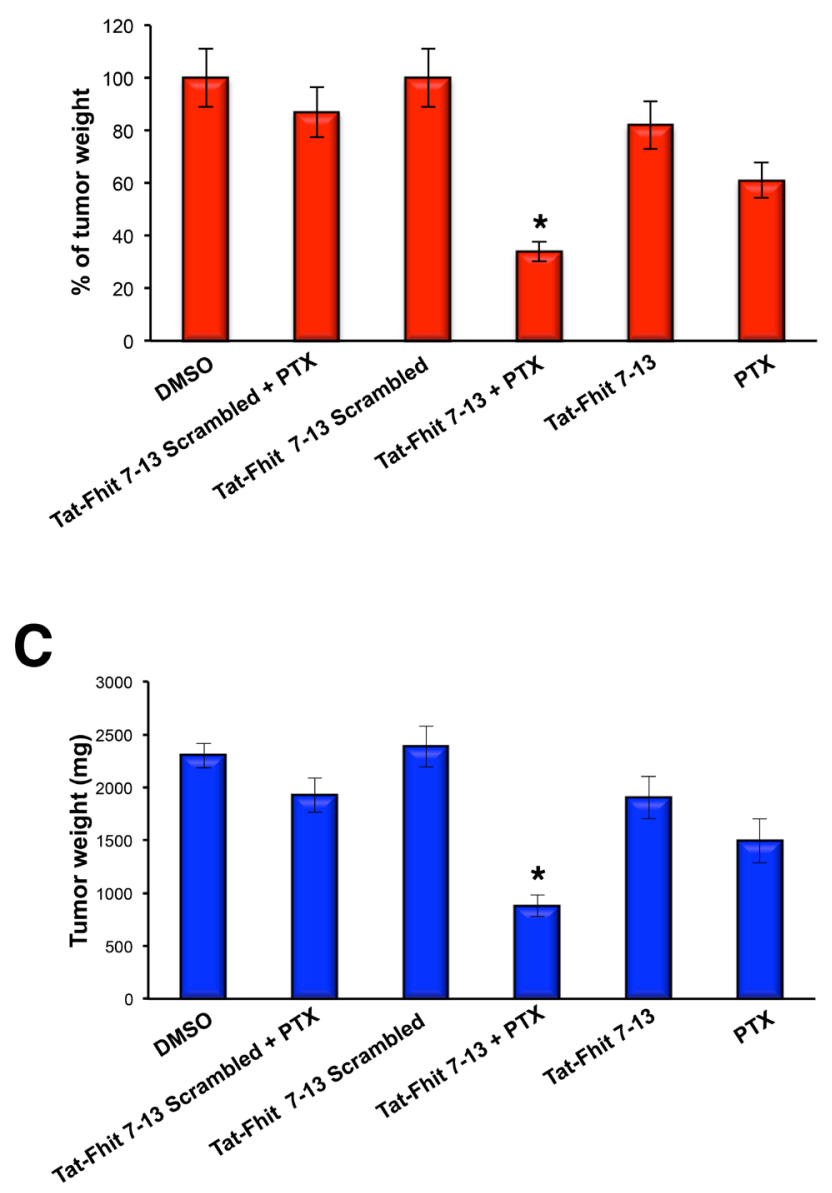

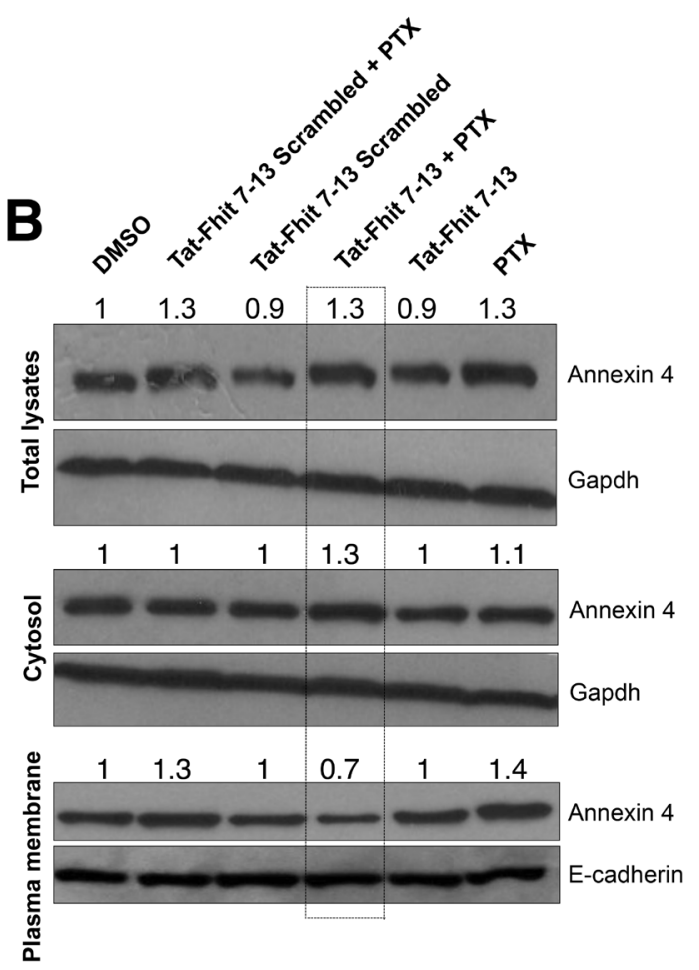

Figure 3: Tat-Fhit 7-13 peptide in combination with paclitaxel blocks in vivo tumor formation. A.,C. Nude mice were subcutaneously injected with $1.5 \times 10^{7}$ A549 cells. When tumors reached $15 \mathrm{~mm}$ diameter, mice were mock-treated, treated paclitaxel (single IV administration of $40 \mathrm{mg} / \mathrm{kg}$ ), with scrambled Tat peptide $(50 \mathrm{mg} / \mathrm{kg}$ ) alone or in combination with paclitaxel, with Tat-Fhit 7-13 peptide $(50 \mathrm{mg} / \mathrm{Kg})$ alone or in combination with paclitaxel; paclitaxel and peptides were injected in a single administration and mice were monitored on a regular basis. Two days later, mice were sacrificed and tumors were evaluated by percent $\mathbf{A}$. and by weight $\mathbf{C}$.. Bar graphs show mean \pm SEM for values from 5 mice $(* P<0,05)$. B. Tumor xenografts excised from mice were lysed, proteins from cytosolic and membrane fractions were separated on a polyacrylamide gel, transferred to nitrocellulose filter, and probed with Annexin 4 antibody. Gapdh and E-cadherin were used to normalize protein loading of cytosolic and plasma membrane proteins, respectively. The numbers above the blots indicate the intensity of the band expressed as ratio "gene product ANNEXIN 4/GAPDH or E-CADHERIN" and normalized to "DMSO". 
plus paclitaxel; $48 \mathrm{~h}$ after treatments, mice were sacrificed and tumors were evaluated by weight. A dramatic effect on tumor growth inhibition was observed in the group of mice treated with both paclitaxel and Tat-Fhit 7-13 peptide compared to Tat-Fhit 7-13 or paclitaxel alone or controls. Tumors treated with Tat-Fhit $7-13$ or paclitaxel showed a reduction in tumor wt to $82 \%$ or $60 \%$ of controls, respectively, while their simultaneous administration resulted in tumor reduction to $33 \%$ of controls (Figure 3A, 3C, and Supplementary Figure S8). After tumor protein lysate fractionation into cytosolic and plasma membrane fractions, we observed that Tat-Fhit 7-13 treatment reduced the extent of Annexin A4 translocation to the plasma membrane after in vivo administration of paclitaxel (Figure 3B), thus indicating that the reported effect on tumor shrinkage in mice treated with the Tat-Fhit 7-13 plus paclitaxel was mainly due to the ability of the peptide to trap Annexin A4 in the cytosol.

\section{DISCUSSION}

Fhit loss has been extensively linked to many processes involved in human and experimental tumorigenesis, including reduced apoptosis, genomic instability, chemoresistance, and, more recently, invasion [29-31], even though the mechanisms governing its activity are still largely obscure. The contribution of Fhit loss to these hallmarks of cancer [19] makes this small protein a very interesting candidate for the development of novel anticancer therapies. However, as no pathway for Fhit function has been discovered for long time after FHIT gene cloning, the only therapeutic approach used to restore Fhit function in cancer cells has been limited to virus-mediated gene therapy of preclinical models of cancer [32].

We recently begun to shed lights about Fhit protein partners unveiling novel pathways depending upon it; in fact, by using a proteomic approach we reported for the first time a Fhit complex including Hsp10, Hsp60, and ferredoxin reductase which underlies and supports the role of Fhit in apoptosis through the generation of free radicals in mitochondria [17]. However, even though there are growing evidences of mitochondria as druggable organelles for cancer therapy [33], Fhit is not suitable to this matter, as it is evident the need of the wild-type protein to restore susceptibility to apoptosis triggered by reactive species of oxygen in Fhit-negative cancer cells $[15,16,34]$. Thus, although encouraging as a proof of principle, the possibility of overexpressing the native Fhit protein through viral vectors is very far from large-scale clinical applications for cancer treatment.

These findings prompted us to search for novel Fhit partners with the purpose to identify proteins with oncogenic activity in order to interfere with their function in cancer cells. The isolation of Annexin A4 as a novel Fhit-interacting protein was a very interesting finding for further studies because of its oncogenic role in the pathogenesis of cancer $[19,28]$ and chemoresistance, assessed for the first time in renal cancer cells resistant to paclitaxel and then also highlighted with cisplatin in cancer cell lines of different types $[35,36]$. Intriguingly, as Fhit loss is also involved in cancer cells refractory to paclitaxel and cisplatin $[17,30]$, the discovery of the Fhit-ANXA4 complex offers opportunities of investigation to search for novel mechanisms responsible for chemoresistance, an event connected to the failure of pharmacological treatment of cancer patients that accounts for their dismal prognosis.

We recently studied the possible role of Fhit in paclitaxel resistance with regard to ANXA4 overexpression; we demonstrated that, while ANXA4 is overexpressed and located to the inner side of plasma membrane in Fhit-negative lung cancer cells treated with paclitaxel, virally-mediated Fhit overexpression restores sensitivity to paclitaxel both in vitro and in vivo by delocalizing ANXA4 to cytosol [19]. In the present study, as apparently the interference with ANXA4 could possibly not require a full length Fhit protein, we explored the opportunity to identify the shortest Fhit protein sequence still able to recognize the oncogenic ANXA4 thus exerting the biological effects of the Fhit wild-type protein.

The molecular mapping of the Fhit protein domain interacting with Annexin A4 led to narrow the interaction domain to aminoacids 7 to 13 of the Fhit protein. Interestingly, the introduction of this peptide into cells did mimic the behavior of wild-type Fhit protein toward Annexin A4; in fact, the administration of a synthetic TatFhit 7-13 peptide to lung cancer cells: a) blocked Annexin A4 translocation from cytosol to plasma membrane; b) acted synergistically with paclitaxel in triggering apoptosis of lung cancer cells; c) induced dramatic regression of A549 tumor xenografts in combination with paclitaxel.

Our model suggests that both Fhit protein and Fhitmimetic peptide could interfere with a protein complex recruited by ANXA4 at the plasma membrane level thus determining its relocalization to cytosol. The clarification of the fine-tuning mechanisms governing the Fhit-ANXA4 binding will be a quite interesting field of investigation for better understanding the role of both Fhit and ANXA4 in chemoresistance.

In conclusion, these findings open the interesting perspective of the generation of an innovative drug to be translated in cancer therapy, as the pursue of a possible approach able to overcome chemoresistance would be desirable to fight otherwise incurable cancers. The Fhit 7-13 peptide might serve as a lead compound for the development of synthetic Fhit-mimetic small molecules with the fascinating perspective of their combination with already commercially available drugs; this approach would apparently be helpful for patient refractory to conventional therapies alone, thus making anticancer therapies less toxic and more effective. 


\section{MATERIALS AND METHODS}

\section{Cell culture and transfection experiments}

A549 cells were maintained at $37^{\circ} \mathrm{C}$ in a humidified atmosphere of $5 \% \mathrm{CO}_{2}$ in the appropriate growth medium with supplements added as recommended.

\section{Immunoblotting, GST pull-down and fractionation analysis}

Total proteins were extracted with Nonidet P40 (NP40) lysis buffer; cytosolic and plasma membrane proteins were extracted using the FractionPREP-cell fractionation system (Biovision). Total lysates with enriched plasma membrane proteins, used for both mass spectrometry and immunoprecipitations analyses, were obtained using Mem-PER Eukaryotic Membrane Protein Extraction Kit (Pierce).

For immunoblotting, proteins $(50 \mu \mathrm{g})$ were separated on polyacrylamide gels and transferred to nitrocellulose filter membranes. Membranes were blocked in 5\% non-fat dry milk, incubated with primary anti-Annexin 4, GAPDH, and E-Cadherin antibodies (Santa Cruz Biotechnology), detected by the appropriate secondary antibodies, and revealed by enhanced chemiluminescence (ECL; Amersham Inc.).

Full-length FHIT cDNA (441 bp) and shorter cDNAs, FHIT-TR1 (332 bp), FHIT-TR2 (212 bp), and FHIT-TR3 (113 bp) containing the common FHIT 5'-end were cloned in the pGEX-2T plasmid, and were expressed in E. coli (BL21).

To clone wild-type FHIT and the truncated forms, the following primers containing a BamHI restriction site were used:

FHIT forward: 5' GGATCCTCGTTCAGATTTGGCCAA-3'

FHIT rev TR1: 5'

GGATCCGTCATTCCTGTGAAAGTCTCCAGCCTT - 3' FHIT rev TR2: 5'-GGATCC-

CCCATGGAAATGTTTTTCCACCACTGT-3' FHIT rev TR3: 5'-GGATCC-CACAAGGACATGT CCTGGTACCACAGGTT-3'

PGEX-2T plasmid, E. coli BL21, and Glutathione Sepharose 4B resin were from Amersham.

\section{TUNEL assay}

A549 cells were assessed for the induction of single strand breaks (indicative of apoptosis) by the terminal deoxynucleotidyl transferase mediated X-dUTP nick end labeling (TUNEL) assay using the in situ cell death detection kit (Boehringer/Roche), according to the manufacturer's recommendations.

\section{Flow cytometry}

A549 cells were collected and washed in PBS solution. DNA was stained with propidium iodide $(50 \mu \mathrm{g} /$ $\mathrm{ml}$ ) and analyzed with a FACScan flow cytometer (BectonDickinson) interfaced a Hewlett-Packard computer. Cell cycle data were analyzed with the CELL-FIT program (Becton-Dickinson).

\section{Fhit peptides}

Tat peptide (from the amino acids 47-58 HIV-1 Tat protein -YGRKKRRQRRR) was synthesized upstream to Fhit peptides in order to drive them into the cell. Both Fhit and scrambled peptides were from Invitrogen. A549 cells were treated with the following peptides: Tat-Fhit 7-13 (Tat-G-QHLIKPS); Tat-Scrambled 7-13 (Tat-GLSKQPHI). Peptides were administered to cell cultures at the concentrations of 50, 100, and 150 microM; $24 \mathrm{~h}$ after treatment, cell cycle perturbations were assessed by flow cytometric analysis $[37,38]$.

\section{Animal studies}

Mice were maintained and animal experiments conducted under institutional guidelines established for the Animal Facility at The Ohio State University; nu/ nu mice were purchased from The Jackson Laboratory. Tumors were established by injecting $1 \times 10^{7}$ A549 cells subcutaneously into the right flanks of 6 wk-old female nude (nu/nu) mice. Paclitaxel was administrated intravenously as a single treatment at the concentration of $40 \mathrm{mg} / \mathrm{kg}$. Synthetic peptides were used in vivo at the concentration of $50 \mathrm{mg} / \mathrm{kg}$; mice were injected intravenously with a single dose of peptides and sacrificed $48 \mathrm{~h}$ later to evaluate tumor weight.

Tumor size was measured on regular basis by using a digital caliper and the equation $\mathrm{V}\left(\mathrm{in} \mathrm{mm}^{3}\right)=\left(\mathrm{A} \mathrm{x} \mathrm{B}^{2}\right) / 2$, where $\mathrm{A}$ is the largest diameter and $\mathrm{B}$ is the perpendicular diameter.

\section{Molecular modeling}

With the aim to identify a target model for our theoretical studies, the 3D crystallographic structures, available into the Protein Data Bank (PDB) with codes 1EMS, 1-2FHI, 1-6FIT [39-43], reporting Fhit, alone or interacting with ligands or proteins, were selected and graphically inspected (Supplementary Figure S1). The model 4FIT, reporting the lowest number of missing residues and a X-ray resolution equal to $2.5 \AA$, was selected and considered in our simulation. The original 
PDB structure required a preliminary treatment consisting in the addition of the missing amminoacid at position 1 and of the highly flexible loop corresponding to the sequence 108-127. Co-crystallized water molecules were removed and hydrogen atoms were added. All preliminary procedures were carried out using the Prime module and the Maestro GUI, as implemented in the Schrodinger software suite 2011 [44]. Shortened Fhit derivatives, sequences 1-38 and 7-13, were obtained from the 4FIT model. All molecular dynamics (MD) simulations were performed using the force field OPLSA-2005, up to $15 \mathrm{~ns}$ at $300^{\circ} \mathrm{K}$ using the version 3.0 of the Desmond program [45] sampling 150 structures at regular time intervals. Water solvent effects were taken into account by means of the explicit solvation model TIP3. Lys sidechains solvent accessible surface areas were computed onto the MD sampled structures considering a water mimicking probe with a radius equal to $1.4 \AA$. MD trajectories were clustered using the average hierarchical linkage method considering a backbone related root mean square deviation (RMSd) cutoff equal to $2.0 \AA$ among the members of the same cluster. The most representative structure, of each cluster, was submitted to quantum mechanics/molecular mechanics (QM/MM) approach for computing the Mulliken charge distribution related to the Lys sidechains. These residues were treated using the density functional theory (DFT) and the LACVP* basis set, the rest of the target was optimized using the OPLS-2005 force field. QM/MM calculations were performed by means of the version 5.7 of the QSite software $[46,47]$.

\section{Statistics}

All graph values represent means \pm SEM from three independent experiments with each measured in triplicate. The differences between two groups were analyzed with unpaired two-tailed Student's $t$ test. $P<0.05$ was considered statistically significant and indicated with asterisks as described in figure legends.

\section{ACKNOWLEDGMENTS}

This work is dedicated to the memory of Pietro Gaudio, Geometra, who died on March 11, 1996. This work was supported by AIRC (Associazione Italiana Ricerca Cancro) (F.T.) and NIH grant CA152758 (C.M.C.). This study was also partly supported by a donation to F.T. in loving memory of Carmelo Stillitani through the FUMG (Fondazione Università Magna Græcia - Catanzaro). The authors are grateful to Dr. Luisa Infante for the manuscript editing.

\section{CONFLICTS OF INTERESTS}

The authors declare that they have no competing financial interests.

\section{Author contributions}

E.G., C.M.C., and F.T. designed the experiments. E.G., F.P., A.N., F.L., N.Z., F.O., S.P., S.D. performed research experiments. R.I.A., S.A. contributed new reagents. E.G., S.A., E.N., P.C., C.M.C., and F.T. analyzed results. E.G., C.M.C. and F.T. wrote the paper. All authors critically reviewed the manuscript and approved the final version.

\section{REFERENCES}

1. Ohta M, Inoue H, Cotticelli MG, Kastury K, Baffa R, Palazzo J, Siprashvili Z, Mori M, McCue P, Druck T, Croce CM and Huebner K. The FHIT gene, spanning the chromosome 3 p14.2 fragile site and renal carcinomaassociated $\mathrm{t}(3 ; 8)$ breakpoint, is abnormal in digestive tract cancers. Cell. 1996; 84:587-597.

2. Matsuyama A, Shiraishi T, Trapasso F, Kuroki T, Alder H, Mori M, Huebner K and Croce CM. Fragile site orthologs FHIT/FRA3B and Fhit/Fra14A2: evolutionarily conserved but highly recombinogenic. Proceedings of the National Academy of Sciences of the United States of America. 2003; 100:14988-14993.

3. Letessier A, Millot GA, Koundrioukoff S, Lachages AM, Vogt N, Hansen RS, Malfoy B, Brison O and Debatisse M. Cell-type-specific replication initiation programs set fragility of the FRA3B fragile site. Nature. 2011; 470:120123.

4. Huebner $\mathrm{K}$ and Croce CM. FRA3B and other common fragile sites: the weakest links. Nature reviews Cancer. 2001; 1:214-221.

5. Huebner K, Garrison PN, Barnes LD and Croce CM. The role of the FHIT/FRA3B locus in cancer. Annual review of genetics. 1998; 32:7-31.

6. Kuroki T, Trapasso F, Yendamuri S, Matsuyama A, Alder $\mathrm{H}$, Mori $\mathrm{M}$ and Croce CM. Allele loss and promoter hypermethylation of VHL, RAR-beta, RASSF1A, and FHIT tumor suppressor genes on chromosome $3 p$ in esophageal squamous cell carcinoma. Cancer research. 2003; 63:37243728 .

7. Zanesi N, Fidanza V, Fong LY, Mancini R, Druck T, Valtieri M, Rudiger T, McCue PA, Croce CM and Huebner $\mathrm{K}$. The tumor spectrum in FHIT-deficient mice. Proceedings of the National Academy of Sciences of the United States of America. 2001; 98:10250-10255.

8. Dumon KR, Ishii H, Fong LY, Zanesi N, Fidanza V, Mancini R, Vecchione A, Baffa R, Trapasso F, During MJ, 
Huebner K and Croce CM. FHIT gene therapy prevents tumor development in Fhit-deficient mice. Proceedings of the National Academy of Sciences of the United States of America. 2001; 98:3346-3351.

9. Ishii H, Zanesi N, Vecchione A, Trapasso F, Yendamuri S, Sarti M, Baffa R, During MJ, Huebner K, Fong LY and Croce CM. Regression of upper gastric cancer in mice by FHIT gene delivery. FASEB journal. 2003; 17:1768-1770.

10. Ishii H, Dumon KR, Vecchione A, Trapasso F, Mimori K, Alder H, Mori M, Sozzi G, Baffa R, Huebner K and Croce CM. Effect of adenoviral transduction of the fragile histidine triad gene into esophageal cancer cells. Cancer research. 2001; 61:1578-1584.

11. Sevignani C, Calin GA, Cesari R, Sarti M, Ishii H, Yendamuri S, Vecchione A, Trapasso F and Croce CM. Restoration of fragile histidine triad (FHIT) expression induces apoptosis and suppresses tumorigenicity in breast cancer cell lines. Cancer research. 2003; 63:1183-1187.

12. Pichiorri F, Trapasso F, Palumbo T, Aqeilan RI, Drusco A, Blaser BW, Iliopoulos D, Caligiuri MA, Huebner K and Croce CM. Preclinical assessment of FHIT gene replacement therapy in human leukemia using a chimeric adenovirus, Ad5/F35. Clinical cancer research. 2006; 12:3494-3501.

13. Barnes LD, Garrison PN, Siprashvili Z, Guranowski A, Robinson AK, Ingram SW, Croce CM, Ohta M and Huebner K. Fhit, a putative tumor suppressor in humans, is a dinucleoside 5',5"'-P1,P3-triphosphate hydrolase. Biochemistry. 1996; 35:11529-11535.

14. Brenner C, Pace HC, Garrison PN, Robinson AK, Rosler A, Liu XH, Blackburn GM, Croce CM, Huebner K and Barnes LD. Purification and crystallization of complexes modeling the active state of the fragile histidine triad protein. Protein engineering. 1997; 10:1461-1463.

15. Trapasso F, Krakowiak A, Cesari R, Arkles J, Yendamuri $\mathrm{S}$, Ishii H, Vecchione A, Kuroki T, Bieganowski P, Pace HC, Huebner K, Croce CM and Brenner C. Designed FHIT alleles establish that Fhit-induced apoptosis in cancer cells is limited by substrate binding. Proceedings of the National Academy of Sciences of the United States of America. 2003; 100:1592-1597.

16. Semba S, Trapasso F, Fabbri M, McCorkell KA, Volinia S, Druck T, Iliopoulos D, Pekarsky Y, Ishii H, Garrison PN, Barnes LD, Croce CM and Huebner K. Fhit modulation of the Akt-survivin pathway in lung cancer cells: Fhit-tyrosine 114 (Y114) is essential. Oncogene. 2006; 25:2860-2872.

17. Trapasso F, Pichiorri F, Gaspari M, Palumbo T, Aqeilan RI, Gaudio E, Okumura H, Iuliano R, Di Leva G, Fabbri M, Birk DE, Raso C, Green-Church K, Spagnoli LG, Venuta S, Huebner K, et al. Fhit interaction with ferredoxin reductase triggers generation of reactive oxygen species and apoptosis of cancer cells. The Journal of biological chemistry. 2008; 283:13736-13744.

18. Bianchi F, Sasso M, Turdo F, Beretta GL, Casalini P, Ghirelli C, Sfondrini L, Menard S, Tagliabue E and
Campiglio M. Fhit Nuclear Import Following EGF Stimulation Sustains Proliferation of Breast Cancer Cells. Journal of cellular physiology. 2015; 230:2661-2670.

19. Gaudio E, Paduano F, Spizzo R, Ngankeu A, Zanesi N, Gaspari M, Ortuso F, Lovat F, Rock J, Hill GA, Kaou M, Cuda G, Aqeilan RI, Alcaro S, Croce CM and Trapasso F. Fhit delocalizes annexin a4 from plasma membrane to cytosol and sensitizes lung cancer cells to Paclitaxel. PloS one. 2013; 8:e78610.

20. Gerke V and Moss SE. Annexins: from structure to function. Physiological reviews. 2002; 82:331-371.

21. Kim A, Enomoto T, Serada S, Ueda Y, Takahashi T, Ripley B, Miyatake T, Fujita M, Lee CM, Morimoto K, Fujimoto M, Kimura T and Naka T. Enhanced expression of Annexin A4 in clear cell carcinoma of the ovary and its association with chemoresistance to carboplatin. International journal of cancer. 2009; 125:2316-2322.

22. Zimmermann U, Balabanov S, Giebel J, Teller S, Junker H, Schmoll D, Protzel C, Scharf C, Kleist B and Walther $\mathrm{R}$. Increased expression and altered location of annexin IV in renal clear cell carcinoma: a possible role in tumour dissemination. Cancer letters. 2004; 209:111-118.

23. Xin W, Rhodes DR, Ingold C, Chinnaiyan AM and Rubin MA. Dysregulation of the annexin family protein family is associated with prostate cancer progression. The American journal of pathology. 2003; 162:255-261.

24. Duncan R, Carpenter B, Main LC, Telfer C and Murray GI. Characterisation and protein expression profiling of annexins in colorectal cancer. British journal of cancer. 2008; 98:426-433.

25. Yamashita T, Nagano K, Kanasaki S, Maeda Y, Furuya T, Inoue M, Nabeshi H, Yoshikawa T, Yoshioka Y, Itoh N, Abe Y, Kamada H, Tsutsumi Y and Tsunoda S. Annexin A4 is a possible biomarker for cisplatin susceptibility of malignant mesothelioma cells. Biochemical and biophysical research communications. 2012; 421:140-144.

26. Choi $\mathrm{CH}$, Sung CO, Kim HJ, Lee YY, Song SY, Song T, Kim J, Kim TJ, Lee JW, Bae DS and Kim BG. Overexpression of annexin A4 is associated with chemoresistance in papillary serous adenocarcinoma of the ovary. Human pathology. 2013; 44:1017-1023.

27. Mogami T, Yokota N, Asai-Sato M, Yamada R, Koizume S, Sakuma Y, Yoshihara M, Nakamura Y, Takano Y, Hirahara F, Miyagi $Y$ and Miyagi E. Annexin A4 is involved in proliferation, chemo-resistance and migration and invasion in ovarian clear cell adenocarcinoma cells. PloS one. 2013; 8:e80359.

28. Wei B, Guo C, Liu S and Sun MZ. Annexin A4 and cancer. Clinica chimica acta. 2015; 447:72-78.

29. Pichiorri F, Palumbo T, Suh SS, Okamura H, Trapasso F, Ishii H, Huebner K and Croce CM. Fhit tumor suppressor: guardian of the preneoplastic genome. Future oncology (London, England). 2008; 4:815-824.

30. Kim CH, Yoo JS, Lee CT, Kim YW, Han SK, Shim YS 
and Yoo CG. FHIT protein enhances paclitaxel-induced apoptosis in lung cancer cells. International journal of cancer. 2006; 118:1692-1698.

31. Joannes A, Bonnomet A, Bindels S, Polette M, Gilles C, Burlet H, Cutrona J, Zahm JM, Birembaut P and NawrockiRaby B. Fhit regulates invasion of lung tumor cells. Oncogene. 2010; 29:1203-1213.

32. Ishii H, Vecchione A, Fong LY, Zanesi N, Trapasso F, Furukawa Y, Baffa R, Huebner K and Croce CM. Cancer prevention and therapy in a preclinical mouse model: impact of FHIT viruses. Current gene therapy. 2004; 4:5363.

33. Weinberg SE and Chandel NS. Targeting mitochondria metabolism for cancer therapy. Nature chemical biology. 2015; 11:9-15.

34. Pichiorri F, Okumura H, Nakamura T, Garrison PN, Gasparini P, Suh SS, Druck T, McCorkell KA, Barnes LD, Croce CM and Huebner K. Correlation of fragile histidine triad (Fhit) protein structural features with effector interactions and biological functions. The Journal of biological chemistry. 2009; 284:1040-1049.

35. Han EK, Tahir SK, Cherian SP, Collins N and Ng SC. Modulation of paclitaxel resistance by annexin IV in human cancer cell lines. British journal of cancer. 2000; 83:83-88.

36. Morimoto A, Serada S, Enomoto T, Kim A, Matsuzaki S, Takahashi T, Ueda Y, Yoshino K, Fujita M, Fujimoto M, Kimura $\mathrm{T}$ and Naka T. Annexin A4 induces platinum resistance in a chloride-and calcium-dependent manner. Oncotarget. 2014; 5:7776-7787. doi:10.18632/ oncotarget.2306.

37. Paduano F, Ortuso F, Campiglia P, Raso C, Iaccino E, Gaspari M, Gaudio E, Mangone G, Carotenuto A, Bilotta A, Narciso D, Palmieri C, Agosti V, Artese A, GomezMonterrey I, Sala $\mathrm{M}$, et al. Isolation and functional characterization of peptide agonists of PTPRJ, a tyrosine phosphatase receptor endowed with tumor suppressor activity. ACS chemical biology. 2012; 7:1666-1676.

38. Ortuso F, Paduano F, Carotenuto A, Gomez-Monterrey I, Bilotta A, Gaudio E, Sala M, Artese A, Vernieri E, Dattilo V, Iuliano R, Brancaccio D, Bertamino A, Musella S, Alcaro S, Grieco P, et al. Discovery of PTPRJ agonist peptides that effectively inhibit in vitro cancer cell proliferation and tube formation. ACS chemical biology. 2013; 8:1497-1506.

39. Bernstein FC, Koetzle TF, Williams GJ, Meyer EF, Jr., Brice MD, Rodgers JR, Kennard O, Shimanouchi T and Tasumi M. The Protein Data Bank: a computer-based archival file for macromolecular structures. Journal of molecular biology. 1977; 112:535-542.

40. Pace HC, Garrison PN, Robinson AK, Barnes LD, Draganescu A, Rosler A, Blackburn GM, Siprashvili $\mathrm{Z}$, Croce CM, Huebner $\mathrm{K}$ and Brenner C. Genetic, biochemical, and crystallographic characterization of Fhitsubstrate complexes as the active signaling form of Fhit. Proceedings of the National Academy of Sciences of the
United States of America. 1998; 95:5484-5489.

41. Pace HC, Hodawadekar SC, Draganescu A, Huang J, Bieganowski P, Pekarsky Y, Croce CM and Brenner C. Crystal structure of the worm NitFhit Rosetta Stone protein reveals a Nit tetramer binding two Fhit dimers. Current biology. 2000; 10:907-917.

42. Lima CD, D’Amico KL, Naday I, Rosenbaum G, Westbrook EM and Hendrickson WA. MAD analysis of FHIT, a putative human tumor suppressor from the HIT protein family. Structure. 1997; 5:763-774.

43. Lima CD, Klein MG and Hendrickson WA. Structure-based analysis of catalysis and substrate definition in the HIT protein family. Science. 1997; 278:286-290.

44. Jacobson MP, Friesner RA, Xiang Z and Honig B. On the role of the crystal environment in determining protein sidechain conformations. Journal of molecular biology. 2002; 320:597-608.

45. Guo Z, Mohanty U, Noehre J, Sawyer TK, Sherman W and Krilov G. Probing the alpha-helical structural stability of stapled p53 peptides: molecular dynamics simulations and analysis. Chemical biology \& drug design. 2010; 75:348359.

46. Philipp D, Friesner RA Mixed ab initio QM/MM modeling using frozen orbitals and tests with alanine dipeptide and tetrapeptide. J Comp Chem 1999; 20:1468-1494.

47. Murphy RB PD, Friesner RA A mixed quantum mechanics/ molecular mechanics (QM/MM) method for large-scale modeling of chemistry in protein environments. J Comp Chem 2000; 21:1452-1457. 